\title{
Parasitic fibroid of greater omentum in 31-years old woman
}

\author{
Magdalena Smyka®, Bronislawa Pietrzak@, Robert Matusiak, Iwona Szymusik® \\ $1^{\text {st }}$ Department of Obstetrics and Gynecology, Medical University of Warsaw, Poland
}

\begin{abstract}
Uterine leiomyomas are the most common benign tumors of the uterine smooth muscle. The latest FIGO classification, considering both their location and the degree of ingrowth into the uterine muscle, distinguishes eight classes of fibroids [1]. The location of leiomyomas in connection with their size may determine the characteristic symptoms: abdominal pain, pressure symptoms, difficulties in getting pregnant, recurrent miscarriages. Among the case reports there are also descriptions of the so-called parasitic leiomyomas [2]. The paper presents a case report of a parasitic leiomyoma in a young woman who has not been operated on in the abdominal or pelvic organs so far.
\end{abstract}

Key words: parasitic leiomyoma; parasitic myoma; parasitic fibroid

Ginekologia Polska 2022; 93, 1: 88-89

\section{CASE REPORT}

The 31-year-old patient was admitted to the Gynecology Department of the $1^{\text {st }}$ Department and Clinic of Obstetrics and Gynecology at the Medical University of Warsaw in June 2021. The patient, previously treated at the Infertility Clinic, was referred for a diagnostic laparoscopy with examination of the patency of the fallopian tubes.

Before admission to the Department, in a gynecological examination, the uterine body was slightly enlarged, mobile, and there were no changes in the reproductive organ. The ultrasound examination showed a normal uterine body of dimensions $38 \times 41 \mathrm{~mm}$, homogeneous endometrium $10 \mathrm{~mm}$, appendages normal on both sides, a solid lesion of approximately $50 \mathrm{~mm}$, without vascular flows, the ultrasound image most likely corresponding to uterine myoma.

The patient qualified for surgical treatment. The laparoscopy revealed a normal, smooth and mobile body of the uterus, ovaries and fallopian tubes on both sides macroscopically normal. Slightly above the bottom of the uterus, there was a tumor with the appearance of a myoma, $5 \times 6 \mathrm{~cm}$ in size, fixed to the greater omentum. In the peduncle, a feeding vessel emerging from the greater omentum was visualized, the lesion had no contact with the uterine muscle (Fig. $1 \mathrm{~A}, \mathrm{~B})$. The tumor was dissected from the omentum, placed in an end bag and after morcellation was taken outside. The lesion had a firm consistency, and in a cross-section with a whitish color, macroscopically clearly indicated the diagnosis of myoma. Moreover, a chromoscopy confirmed the patency of both fallopian tubes.

The patient was discharged home without any complaints the next day after the surgery. The histopathological examination confirmed the diagnosis of uterine leiomyoma.

\section{DISCUSSION}

Parasitic leiomyomas are extremely rare. So far, the literature has described the occurrence of primary tumors that have acquired new vascularization from organs other than the uterine muscle, and iatrogenic tumors resulting from previously performed laparoscopic procedures $[3,4]$. In times of widespread ultrasonography, the diagnosis of uterine leiomyomas is usually not difficult and their suspicion in ultrasound is most often confirmed by the histopathological result. In 2016. Pradip described the case of a 31-year-old female patient with a $30 \mathrm{~cm}$ tumor filling the abdominal cavity, which turned out to be a parasitic myoma pedunculated to the greater omentum [5]. Although

1st Department of Obstetrics and Gynecology, Medical University of Warsaw, Poland, 1/3 Starynkiewicza St, 02-015 Warsaw, Poland

e-mail: magda.smyka@gmail.com 


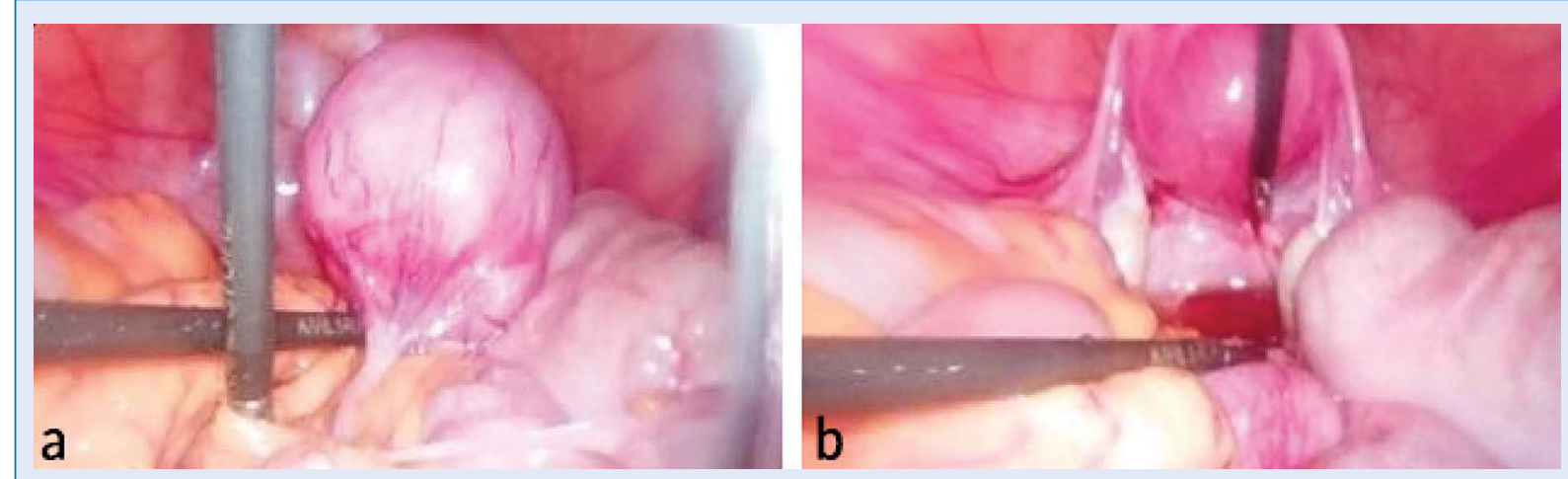

Figure 1. A. - laparoscopy photo: parasitic myoma fixed to the greater omentum, the peduncle is visible; B. - laparoscopy photo: uterus, fallopian tubes and ovaries; some fluid visible in rectouterine pouch

they have been described so far as casuistic cases, it is worth noting the possibility of their occurrence, especially in the era of the current development of laparoscopic techniques.

\section{Conflict of interest}

All authors declare no conflict of interest.

\section{REFERENCES}

1. Munro MG, Critchley HOD, Broder MS, et al. FIGO Working Group on Menstrual Disorders. FIGO classification system (PALM-COEIN) for causes of abnormal uterine bleeding in nongravid women of reproductive age. Int J Gynaecol Obstet. 2011; 113(1): 3-13, doi: 10.1016/j.ijgo.2010.11.011, indexed in Pubmed: 21345435.

2. Sidorowicz M, Nowosielski K, Kędziora H, et al. Peritoneal parasitic myoma in a patient without previous surgery on the reproductive organ - a case report. GinPolMedProject. 2016; 3(41): 85-87.

3. Oindi FM, Mutiso SK, Obura T. Port site parasitic leiomyoma after laparoscopic myomectomy: a case report and review of the literature. J Med Case Rep. 2018; 12(1): 339, doi: 10.1186/s13256-018-1873-y, indexed in Pubmed: 30428912.

4. Anfinan NM. latrogenic parasitic leiomyoma after robotic surgery, managed during cesarean section. Case Report. Journal of Pregnancy and Reproduction. 2018; 2(6), doi: 10.15761/jpr.1000153.

5. Dashraath P, Lim LiM, Huang Z, et al. Parasitic leiomyoma. Am J Obstet Gynecol. 2016; 215(5): 665.e1-665.e2, doi: 10.1016/j.ajog.2016.07.017, indexed in Pubmed: 27430586. 